\title{
FACTORES QUE HAN INFLUIDO EN LA DETERMINACION DE LA TASA SALARIAL EN EL MERCADO DE TRABAJO MEXICANO
}

\author{
José Raúl Luyando Cuevas ${ }^{1}$
}

\begin{abstract}
Resumen
El documento analiza los factores que han influido en el salario de los trabajadores mexicanos antes de la apertura comercial y en la primera y segunda etapa de la apertura comercial mexicana. Contrario a muchas de las investigaciones realizadas sobre este fenómeno, el presente trabajo encuentra que en promedio la escolaridad no tiene un efecto relevante sobre el salario de los trabajadores en la primera y segunda etapa de la apertura comercial.
\end{abstract}

Clasificación JEL: C21 - J24 - J31

Palabras clave: trabajo - mercado - salarios

\begin{abstract}
The paper analyzes the factors that have influenced the wages of Mexican workers before the Commercial Opening and the first and second stages of the Mexican trade liberalization. Unlike many of the research on this phenomenon, this paper finds that the average schooling has no significant effect on wages of workers in the first and second stages of trade liberalization.
\end{abstract}

JEL-Classification: C21 - J24 - J31

Keywords: labor - market - wages

\footnotetext{
${ }^{1}$ Estancia posdoctoral en la Universidad Autónoma Metropolitana Iztapalapa, en el Doctorado en Estudios Sociales. Agradezco el apoyo que recibí de CONACYT para realizar la presente investigación, e.mail: jrlc9@hotmail.com.
} 


\section{INTRODUCCION}

El interés de numerosas investigaciones sobre los factores que han influido en la tasa salarial de los trabajadores en el mercado laboral mexicano, se hace desde la perspectiva de una apertura comercial vertiginosa -el índice de apertura comercial pasa de $26.64 \%$ en el año de 1984 a ser de $86.81 \%$ en el año de $1999^{2}$-, que inicia el gobierno mexicano en los años ochentas. La apertura comercial, en este sentido, afectaría de manera casi inmediata a los procesos productivos del país, y por tanto, al factor laboral. La lógica económica ortodoxa, nos indicaría que los países no desarrollados tenían una ventaja comparativa con respecto a los países desarrollados, su abundante oferta de trabajadores con bajos niveles de cualificaciones. En este sentido, muchos procesos productivos que requerían de trabajadores con este tipo de características, se trasladarían de países desarrollados a países no desarrollados, buscando reducir sus costos de producción. Bajo esta perspectiva, los trabajadores, en países no desarrollados, con bajas cualificaciones se beneficiarían de la apertura comercial emprendida por sus respectivos gobiernos, en dos sentidos: por un lado, encontrando puestos laborales donde ocuparse, y por otro, al ver incrementarse sus salarios, por una mayor demanda. El problema con esta lógica, fue que muchas

${ }^{2}$ Luyando, J. R., (2007), Ensayos Sobre Mercados de Trabajo Duales: El Caso Mexicano, Tesis Doctoral, Departamento de Fundamentos del Análisis Económico, Historia e Instituciones Económicas, Universidad de Valladolid, p. 102. 
investigaciones encontraron evidencia de que se había incrementado la desigualdad salarial en los países no desarrollados, es decir, habían encontrado que los salarios de los trabajadores con mayores cualificaciones se habían incrementado en relación con los salarios de los trabajadores con bajas cualificaciones. Había ocurrido una situación contraria a la pronosticada y muy similar a la que sucedió en los países desarrollados. Con la salvedad de que la desigualdad salarial en los países no desarrollados antes de la apertura comercial, era muy superior a la que podríamos encontrar en los países desarrollados.

Autores como Feliciano (2001), Hanson (1995, 2003 y 2005) y Cragg y Epelbaum (1996) encontraron que la dispersión salarial en México se incrementó dada la política de apertura comercial llevada a cabo por el gobierno. Estas investigaciones, en general, hablan del incremento en los rendimientos sobre años de escolaridad respecto a los salarios que obtuvieron los trabajadores mexicanos. En este sentido, Robertson (2001), señala que el retorno sobre escolaridad, para trabajadores urbanos, aumentó de 0.035 en 1987 a 0.05 en 1994 y 0.07 en 1998.

Como era de esperarse, lo siguiente que se preguntaron muchos de estos y otros investigadores, eran las causas de este fenómeno. Una explicación casi intuitiva, es que, la demanda de trabajadores con mayores cualificaciones aumento con la apertura 
comercial $^{3}$. Autores como Cragg y Epelbaum (1996), sugieren que esta es la causa, por la que, están aumentando los salarios de los trabajadores con mayor escolaridad. Pero surge la pregunta de ¿qué elementos fueron los causantes del aumento en la demanda? Algunos autores, como Robertson (2001), por lo regular basados en el Teorema de Stolper-Samuelson (1941), señalan que durante el periodo, en que, se llevo a cabo el modelo de sustitución de importaciones, los sectores más protegidos fueron los intensivos en trabajo, en su mayoría, con trabajadores con bajas cualificaciones ${ }^{4}$. Observando que estos sectores fueron los más afectados con la apertura comercial, por lo que, los precios de los productos que utilizaban trabajadores con mayores cualificaciones aumentaron en relación a los productos que utilizaban trabajadores con menores cualificaciones. El cambio en precios propició que se redujera la demanda de trabajo en los sectores que requerían de trabajadores con menores cualificaciones y aumentara en las que requerían de trabajadores con mayores

\footnotetext{
3 La mayor demanda de este tipo de trabajadores en un mercado de trabajo como el mexicano, podría propiciar que en el corto plazo sus salarios aumenten, al haber relativamente pocos trabajadores con estas características.

4 Antes de 1980, los esfuerzos de los gobiernos mexicanos estaban enfocados en el llamado modelo de sustitución de importaciones. Este modelo había producido algunos de los resultados que de él se esperaban, pero a costa de la protección férrea de las fuentes de empleo, es decir, comprendía una política de empleo implícita en la protección a las empresas, la promoción de actividades productivas de pequeña escala, e intervención casuística en defensa de fuentes de trabajo. La motivación era la defensa y promoción de puestos de trabajo, dejando, en segundo término, cuestiones de viabilidad económica de las empresas y del conjunto de la economía. Crecientes transferencias gubernamentales sostenían o rescataban de la quiebra a empresas empleadoras de mano de obra.
} 
cualificaciones. Autores como Revenga (1997) y

Feliciano (2001), en general, encuentran que la desigualdad salarial aumenta más en las industrias dedicadas a producir bienes de exportación, y que, los salarios de los trabajadores caen en aquellas industrias que producen bienes para el mercado interno. Otros autores como Feenstra y Hanson (1996), muestran que la demanda de trabajadores con mayores cualificaciones aumentó más en la región fronteriza de México con los Estados Unidos de Norteamérica (E.U.), a donde se dirigió mucha de la inversión extranjera y en donde se establecieron muchos de los procesos productivos de empresas estadounidenses, las llamadas maquiladoras ${ }^{5}$. En este sentido, se habla de una desigualdad regional, pues los estados que en mayor medida se beneficiaron, fueron los fronterizos con E.U. y los que contaban con los más sofisticados medios de comunicación, es decir, con buen acceso a los mercados internacionales ${ }^{6}$.

Por su parte, Hanson y Harrison (1999), sugieren que lo mismo que le pasó a E.U. con México, le pasó a México con países como China. Es decir, la amplia oferta de trabajadores con bajas cualificaciones que tenía México con respecto a E.U. a su vez, la tenían países como China con respecto a México. En este sentido, los procesos productivos que requerían de una abundante

\footnotetext{
${ }^{5}$ Son plantas que importan todos sus componentes de la empresa matriz en los E.U. y ensamblan el producto final en México, para posteriormente vender la mayoría de su producción en los mismos E.U.

${ }^{6}$ Urciaga y Almendarez (2006), determinan en un estudio para la región del mar de Cortés (en el noroeste de la república mexicana), que hay un patrón territorial claramente definido, en el sentido que los trabajadores que habitan en ciudades con mayor desarrollo en la frontera con E.U. reciben mayores salarios.
} 
oferta de trabajadores poco cualificados, se trasladarían a países como China -buscando reducir sus costos de producción- y no a México, por lo que, en México se tendrían que llevar a cabo procesos productivos un poco más refinados o que requerían de un tipo de trabajadores más cualificados. De ahí que, la demanda de este tipo de trabajadores aumentaría y la demanda de trabajadores menos cualificados disminuiría, y con ello, sus salarios.

Por último, cabe señalar que en muchos trabajos se habla de que la apertura comercial no fue el único factor que contribuyo a incrementar la desigualdad salarial en México. De hecho, Feliciano (2001), señala que la apertura comercial tuvo un modesto impacto en el mercado de trabajo mexicano y que la desigualdad salarial en mayor medida se debe al programa de estabilización económica, mejor conocido como el Pacto de Solidaridad Económica. Un acuerdo que negoció el gobierno mexicano con representantes de los principales sindicatos, con representantes de las principales uniones agrícolas y con los representantes del sector empresarial, con el objetivo de amarrar las negociaciones salariales a la tasa de inflación esperada anual ${ }^{7}$. También en este

\footnotetext{
${ }^{7}$ El Pacto de Solidaridad Económica se firma el 15 de Diciembre de 1987 y su características principales son:

a. El gobierno se compromete a: reducir su gasto programable de 1988 de 22 a $20.5 \%$ con relación al PIB; aumentar su superávit primario de 5.4 a $8.3 \%$, también en relación al PIB; suprimir subsidios; reducir más el sector paraestatal y racionalizar sus estructuras administrativas.

b. El Congreso del Trabajo declina su reclamo de aumento salarial de emergencia, aceptando la exhortación al sector privado de aumentar el 15\% de emergencia a salarios mínimos y contractuales, y el $20 \%$ en enero sólo a los mínimos. Con la promesa que desde marzo se ajustarán mensualmente, de acuerdo a la canasta básica recientemente anunciada.
} 
sentido, señala la política de reducir los salarios mínimos, que llevo a cabo el gobierno mexicano desde los años setentas.

Por tanto, el interés del presente trabajo, es contribuir a la anterior discusión desde una perspectiva diferente, pero retomando algunas de las contribuciones hechas por las investigaciones anteriores. De estas investigaciones, se puede desprender que el nivel escolar de los trabajadores esta positivamente relacionado con su nivel salarial, y que factores como el sectorial y el regional inciden en los niveles salariales con la apertura comercial. Otros atributos que consideramos influyen en la tasa salarial y que se han incluido en trabajos como el de Urciaga y Almendarez (2006), son el género y el tipo de contratación. El género se introduce para observar, si la condición de género, influye sobre el nivel salarial, como se ha hecho en otras investigaciones, y si es el caso, ver si la apertura comercial ha influido para disminuir esa discriminación laboral. En el caso del tipo de contratación, el mercado laboral mexicano se puede dividir entre trabajadores que tienen un contrato por escrito y los trabajadores que no lo tienen. A grandes rasgos, podríamos decir que los trabajadores con contrato por escrito, son trabajadores formales, pues son trabajadores, que en general, reciben las prestaciones establecidas en las leyes laborales mexicanas. Por el

c. Los campesinos aceptan mantener sus productos a los precios de garantía reales estipulados en 1987.

d. Los empresarios se obligan a aumentar la oferta de productos básicos, a elevar la eficiencia de la planta productiva y a "moderar la variación de todos los precios tomando en cuenta los márgenes de comercialización". 
contrario, los trabajadores sin contrato, en general, serían trabajadores informales, al no recibir las prestaciones establecidas en las leyes laborales mexicanas. Con esta variable queremos captar si la condición laboral (tipo de contrato) influye sobre el nivel salarial de los trabajadores -un aspecto primordial en el mercado de trabajo mexicano, pues desde la apertura comercial, se habla de la importancia creciente que fue adquiriendo la informalidad-. De hecho, Urciaga y Almendarez (2006), encuentran que para la región mar de Cortés, los trabajadores asalariados reciben en promedio mayores salarios que los trabajadores a destajo o por cuenta propia.

Por otra parte, otros atributos que consideramos pueden influir en la tasa salarial son los estudios técnicos y la pertenencia o no del trabajador a un sindicato. En el caso de la educación técnica, el gobierno mexicano tenía un enorme interés en la vinculación de la escolaridad y el puesto laboral. Y uno de los ejes para hacerla, era la educación técnica. En 1978, por decreto presidencial se crearon los CONALEP y con el tiempo otras escuelas similares. Escuelas cuyo objetivo principal se orientó a la formación de profesionales técnicos, egresados de secundaria, que en un principio se pensaron como estudios terminales, con años de escolaridad similares a lo que en México se conoce como Bachillerato. En 1993, como parte de la reforma educativa, el gobierno instrumenta proyectos para la modernización de la educación técnica y la capacitación, con lo cual, se abren más expectativas en materia de capacitación laboral, vinculación intersectorial, apoyo comunitario y asesoría 
y asistencia tecnológicas a las empresas. Es por ello, que pensamos es importante incluirla y ver el efecto que tiene sobre el salario de los trabajadores que cuentan con esta clase de estudios. La variable de pertenencia o no a un sindicato, se incluye en el sentido de tener en cuenta, lo que anteriormente se señaló, de que los sindicatos aceptaron ligar sus negociaciones salariales a la inflación esperada, mediante el Pacto de Solidaridad Económica. Con esto, pensamos los sindicatos, perdieron su principal razón de ser, pues una de las principales (sino es que la principal) labor de un sindicato es defender el poder adquisitivo de sus agremiados, mediante la negociación salarial con el patrón. En éste caso delegaba esta función al gobierno, pues la mayoría de los sindicatos aceptan como válida la cifra de inflación esperada que el mismo gobierno elabora y que los sindicatos acatan como la medida para aumentar los salarios de sus agremiados desde 1987. En este sentido, pensamos que la condición de ser un trabajador sindicalizado, pierde la importancia que antes tenía, como uno de los factores que influían positivamente su salario.

En el caso de las variables educación técnica y sindicato, no podemos decir que somos los primeros en incluirlas para el caso mexicano, pues aunque en la literatura que hemos revisado no se incluyen, la literatura sobre este tema es bastante extensa y podríamos estar cometiendo una imprudencia. Pero el último atributo que incluimos, pensamos es novedoso en algún sentido. Este atributo tiene que ver con la evidencia que encontraron las investigaciones arriba mencionadas $\mathrm{y}$ algunos 
indicios que encontramos al analizar los datos de las muestras utilizadas en el presente trabajo. La evidencia indica que con la apertura comercial aumento la demanda hacia trabajadores con mayores niveles escolares -la mayor cualificación, como se indica arriba, estará estrechamente relacionada con la educación adquirida por el trabajador-. Si tenemos en cuenta que en México la educación se divide entre lo que se denomina educación básica y lo que podríamos denominar educación superior a la básica ${ }^{8}$. Podemos suponer que la demanda se está trasladando a los trabajadores con educación superior a la básica. Con esta variable tratamos de identificar si se está dando una división en este sentido en el mercado de trabajo mexicano.

Por tanto, el objetivo del trabajo será ver cómo influyen estas variables en los salarios de los trabajadores mexicanos antes de la apertura comercial y como se ha modificado esta relación durante la primera y segunda etapa de la apertura comercial mexicana. Para hacerlo se utilizaron las Encuestas Nacionales de Ingresos Gastos de los Hogares (ENIGH), elaboradas en México por el Instituto Nacional de Estadística Geografía e Informática, de los años 1984, 1992 y 2000. La encuesta de 1984, proporciona un panorama de las características de los trabajadores y del mercado laboral antes del ingreso formal de México al GATT -en 1985,

\footnotetext{
${ }^{8}$ La educación básica u obligatoria antes de 1993 era de seis años, mejor conocida como la educación primaria. Con la reforma de 1993, en la educación básica u obligatoria se incluye la educación secundaria, con lo que este tipo de educación pasa de seis a nueve años. Por tanto, la educación no básica consistiría de la educación pre-universitaria (bachillerato, de tres años), la educación universitaria y los posgrados.
} 
México ingresa al Acuerdo General Sobre Aranceles Aduaneros y Comercio (GATT), en donde el gobierno mexicano se compromete a rebajar los aranceles y a eliminar barreras no arancelarias ${ }^{9}$. Terminando así con cuatro décadas de industrialización, mediante el modelo de sustitución de importaciones. En 1989, el gobierno mexicano modera las restricciones para extranjeros, sobre posesión de activos en territorio nacional (se eliminaron las restricciones a la inversión extranjera)-. La encuesta de 1992, nos da la posibilidad de ver como cambiaron o no, esas características cuando México ingresa al GATT (antes de que entrara en vigor el Tratado de Libre Comercio de Norteamérica) -el primero de Enero de 1994, entra en vigor el Tratado de Libre Comercio para América del Norte (TLC), suscrito por los Jefes de Estado de México, Canadá y Los Estados Unidos de Norteamérica y ratificado por los órganos legislativos de los tres países. Con este tratado se consolidan y extienden las reformas de 1985 y 1989-. La encuesta del 2000, un año donde se habían consolidado los efectos de la entrada de México al GATT y en donde pensamos, ya también se habían consolidado algunos de los efectos del TLC, a seis años de su implementación. Este año nos da la posibilidad de contrastar, lo que sucedía en 1984, lo que sucedía en 1992, con lo que sucede en el año $2000^{10}$.

9 El gobierno mexicano protegió, durante décadas, a los artículos producidos al interior de su economía mediante el uso de tarifas y permisos de importación. Las tarifas incrementaban el precio de importación de los artículos, mientras que los permisos limitaban el número de artículos importados.

${ }^{10}$ Según el Instituto Nacional de Estadística Geografía e Informática (quien elabora estas encuestas), las tres encuestas eran compatibles en sus diseños. 


\section{MODELO}

Para realizar la investigación se utiliza el modelo básico de Jacob Mincer $(1974)^{11}$. En este modelo también se consideran los efectos que la capacitación general tiene sobre el salario del trabajador. En este sentido, la teoría del capital humano asume que un mayor capital humano se puede acumular al incrementarse la experiencia laboral del trabajador. Sin embargo, el capital humano también se puede depreciar por el desempleo, la obsolescencia de habilidades y el paso del tiempo (entre mayor sea la edad del trabajador, su capital humano podrá depreciarse, en el sentido que al trabajador le tome un mayor tiempo ejecutar tareas). Para tener en cuenta estos dos efectos, se introducen en el modelo la experiencia y la experiencia al cuadrado. Además, añadimos las variables antes señaladas: sexo, educación técnica, condiciones laborales, la pertenencia o no a un sindicato, sector productivo en donde labora, región geográfica en donde labora y la variable que utilizamos para ver si el mercado laboral se puede dividir entre trabajadores con estudios básicos y trabajadores con estudios superiores a los básicos.

Por tanto, el modelo propuesto, se puede formular de la siguiente manera:

Debemos señalar que esto no sucedía con las encuestas anteriores a 1984, razón para no haber realizado un análisis más extenso, en el que se tuvieran en cuenta esos años

11 Mincer, Jacob (1974), "Schooling, Experience and Earnings", Columbia University Press for National Bureau of Economics Research, New York. 


$$
\begin{aligned}
\ln Y_{i}= & \ln Y_{0}+\beta_{1} \text { Escol }_{i}+\beta_{2} \text { Exp }_{i}+\beta_{3}{\text { Exp } 2_{i}+\beta_{4} \text { Sexo }_{i}+\beta_{5} D_{-} \text {Ed }_{-} \text {Téenica }_{i}} \\
& +\beta_{6} D_{-} \text {Contrato }_{i}+\beta_{7} \text { Sindicato }_{i}+\beta_{8} D_{-} \text {Sector }_{-} I I_{i}+\beta_{9} D_{-} \text {Sector }_{-} I I I_{i} \\
& +\beta_{10} D_{-} \text {Estados_Ind }_{i}+\beta_{11} D_{-} \text {Estados_Fron }_{i}+\beta_{12} D_{-} \text {Ed_Formal }_{i}+u_{i}
\end{aligned}
$$

Donde el significado de cada una de las variables, es el siguiente:

$\ln Y=$ Logaritmo natural del salario por hora.

Escol = Nivel de instrucción en educación básica, media, superior y posgrado.

$\operatorname{Exp}=$ Experiencia.

Exp2 $=$ Experiencia al cuadrado.

Sexo = El género del trabajador, como variable dicotómica.

D_Ed_Técnica $=$ Educación técnica, como variable dicotómica.

D_Contrato $=$ Contrato laboral, como variable dicotómica.

Sindicato $=\mathrm{La}$ afiliación o no de un trabajador a un sindicato, como variable dicotómica.

D_Sector_II = Sector secundario, como variable dicotómica.

D_Sector_III = Sector terciario, como variable dicotómica. 
D_Estados_Ind $=$ Estados con mayor desarrollo industrial, como variable dicotómica.

D_Estados_Fron $=$ Estados fronterizos, como variable dicotómica.

D_Ed_Formal $=$ Variable que nos sirve para dividir a los trabajadores, entre aquellos que cuentan con estudios de educación básica y los que tienen estudios mayores a los básicos, como variable dicotómica. $\mathrm{u}=$ Termino aleatorio.

Si esta última ecuación se ajusta por mínimos cuadrados ordinarios $(\mathrm{MCO})$, la pendiente $\left(\beta_{1}\right)$, nos dará una estimación de la tasa de retorno de la escolaridad y la ordenada al origen, el nivel predecible del logaritmo de salario en ausencia de escolaridad. Se debe hacer notar que la tasa de retorno estimada por este medio, ignora otros gastos o costos que se hacen en el periodo donde el individuo permanece en la escuela y también que el retorno así estimado es privado, ya que omite subsidios a la educación y los posibles beneficios que tiene un individuo más informado para la sociedad.

Sin embargo, cuando se ejecuta la ecuación minceriana mediante MCO, autores como Griliches (1977), señalan que hay un problema en este procedimiento. En términos generales, mencionan que la ecuación no toma en cuenta la habilidad o capacidad innata de los individuos. La inclusión de esta variable implica que dos individuos con idéntico nivel educativo 
y experiencia, pero con distintas capacidades $\mathrm{o}$ habilidades, obtendrán salarios diferentes. Por tanto, la no inclusión de esta variable generaría sesgo en los rendimientos a la escolaridad- sesgo que podría trasladarse a otras variables del modelo-. El principal inconveniente para obtener una solución óptima a este problema, es que, la variable capacidad o habilidad es una variable no observable, pues su significado tiene que ver con inteligencia, talento, aptitud, competencia y destreza, variables que son difíciles de medir. Por tanto, la mayoría de las soluciones que se han propuesto, pasan por hacer alguna aproximación de cualquiera de estas definiciones. Quizá para el caso mexicano, el trabajo de Barceinas (2003), es de los más completos para tratar de explorar este tipo de problema, señalando que: "el estimador por variables instrumentales no representa un rendimiento promedio de la educación, sino el de un grupo particular muy alejado del promedio muestral, pero frecuentemente correlacionado con los instrumentos utilizados. Los instrumentos actúan de forma diferencial sobre grupos que comparten los mismos beneficios y costos marginales y el rendimiento estimado es un promedio de tales efectos" $"$.

En nuestro caso, estamos interesados en el promedio muestral, pues lo que queremos es compararlo contra el que se dio en la primera y segunda etapa de la apertura comercial -y así lo queremos con cada una de las variables del modelo-. Por tanto, más que utilizar los MCO directamente en cada año estudiado, lo que

12 Barceinas, Fernando (2003), "Endogeneidad y Rendimientos de la Educación”, Estudios Económicos 35, Vol. 18, № 1, Enero-Junio, p. 110. 
hacemos es una combinación de cortes transversales independientes. "Al combinar muestras aleatorias extraídas de la misma población, pero en distintos puntos del tiempo, obtenemos estimadores más precisos y estadísticos de prueba con más potencia" ${ }^{13}$. Por lo que, el modelo como combinación de cortes transversales quedaría de la siguiente manera:

$\ln Y_{i}=\ln Y_{0}+\delta_{0} 1992+\gamma_{0} 2000+\beta_{1}$ Escol $+\delta_{1}$ Escol $1992+\gamma_{1}$ Escol $2000+\beta_{2} \operatorname{Exp}$

$+\delta_{2} \operatorname{Exp} 1992+\gamma_{2} \operatorname{Exp} 2000+\beta_{3} \operatorname{Exp} 2+\delta_{3} \operatorname{Exp} 2 \rightarrow 1992+\gamma_{3} \operatorname{Exp} 2 \rightarrow 2000+\beta_{4} \operatorname{Sexo}$

$+\delta_{4}$ Sexo1992 $+\gamma_{4}$ Sexo $2000+\beta_{5} D_{-} E d_{-}$Técnica $+\delta_{5} E d_{-}$Técnica 1992

$+\gamma_{5} E d_{-}$Técnica $2000+\beta_{6} D \_C o n t r a t o+\delta_{6} D \_C o n t r a t o 1992$

$+\gamma_{6} D \_$Contrato $2000+\beta_{7}$ Sindicato $+\delta_{7}$ Sindicato $1992+\gamma_{7}$ Sindicato 2000

$+\beta_{8} D$ _Sector_II $+\delta_{8} D$ _Sector_II $1992+\gamma_{8} D_{-}$Sector_II 2000

$+\beta_{9} D \_S e c t o r \_I I I+\delta_{9} D \_S e c t o r \_I I I 1992+\gamma_{9}$ D_Sector_III 2000

$+\beta_{10} D \_$Estados_Ind $+\delta_{10}$ D_Estados_Ind1992 $+\gamma_{10} D \_$Estados_Ind 2000

$+\beta_{11} D_{-}$Estados_Fron $+\delta_{11} D_{-}$Estados_Fron $1992+\gamma_{11} D_{-}$Estados_Fron 2000

$+\beta_{12} D_{-} E d_{-}$Formal $+\delta_{12} D_{-} E d_{-}$Formal1992 $+\gamma_{12} D_{-} E d_{-}$Formal $2000+u$

Como se puede observar el año base es 1984 (antes de la apertura comercial) y se introducen las variables 1992 y 2000, como variables dicotómicas, para diferenciar estos años del año base.

\section{CARACTERISTICAS DE LAS BASES DE DATOS Y DEFINICION DE LAS VARIABLES UTILIZADAS}

13 Wooldridge, Jeffrey M., Introducción a la Econometría: Un Enfoque Moderno, Thomson Learning, México, 2002, p. 409. 
Como señalamos antes, para elaborar los modelos se utilizaron las Encuesta Nacional de Ingreso y Gasto de los Hogares (ENIGH) de los años de 1984, 1992 y 2000. Con el propósito de crear la base que necesitábamos, utilizamos sólo dos de las bases que se encuentran en la encuesta, la de Ingresos, que detalla el monto de los ingresos y de donde provienen y la llamada Person, donde se detallan las características escolares, sociales y laborales de los miembros del hogar. Estas dos bases se fusionaron en una sola base mediante dos campos comunes, el campo llamado FOLIO, el cual, es un identificador único del hogar y el campo NUMREM, el cual señala el número de renglón del residente del hogar ${ }^{14}$.La intención al fusionarlas, era tener identificadas a las personas que dentro de la familia obtenían algún ingreso, saber cuál era el monto del ingreso, de donde provenía y cuáles eran sus características sociales y laborales.

En los tres años utilizamos lo que se denomina en las encuestas "ingreso mes pasado", es decir, el último ingreso que percibió el trabajador. Tomando sólo los ingresos netos por remuneraciones al salario, es decir, las percepciones que en efectivo obtienen los miembros de hogar a cambio de la venta de su fuerza de trabajo a

\footnotetext{
14 Para realizar la fusión se utilizo el programa Acces. Cabe señalar que para los años 1984 y 2000 no hubo mayor problema. Pero en el año de 1992, se tuvieron varios problemas que se debieron superar. En primer lugar, hay una buena cantidad de trabajadores que no tenían el campo NUMREM, después de intentar varias soluciones, se decidió dejarlos fuera, por considerar era la solución más indicada. En segundo lugar, varios campos estaban aglutinados en uno solo, por lo que se procedió a dividirlos, por medio de la longitud de cada uno de los campos aglutinados, esto se realizo programando en el mismo Acces.
} 
una empresa, institución o patrón, con quién han establecido determinadas condiciones de trabajo mediante un contrato o acuerdo -en especifico hablamos de los sueldos, salarios o jornal y las horas extras ${ }^{15}$. Pero como en el modelo lo que utilizamos como variable dependiente son los logaritmos naturales de salarios por hora, nos servimos de la variable horas trabajadas ${ }^{16}$, para generar esa variable mediante operaciones matemáticas básicas, para posteriormente obtener el logaritmo natural de cada uno de ellos.

Como también ya lo señalamos las variables independientes son: escolaridad, experiencia, experiencia al cuadrado, sexo, educación técnica, rama, tipo de contratación, afiliación a algún sindicato, la entidad federativa y la denominada educación formal ${ }^{17}$. La variable experiencia, la obtenemos de la manera tradicional, es decir, la edad menos los años de educación escolarizada menos seis. La variable experiencia al cuadrado, como su nombre lo indica es la variable experiencia elevada al cuadrado. Para identificar el efecto que tiene el sexo en los salarios por hora, se

\footnotetext{
15 Cuando el trabajador indicaba que tenía más de un ingreso por este mismo rubro, se sumaron estos ingresos y se tomaron como uno sólo, para no tener duplicidad de trabajadores. Es decir, si identificábamos, por medio del número de renglón del residente del hogar, a alguna persona con dos ingresos o más, acumulábamos esos ingresos, en el sentido de no crear distorsiones en el interior de la muestra, esto se realizó por medio del programa Crystal Reports.

${ }^{16}$ En caso de que algún trabajador señalará tener más de un trabajo, las horas de todos sus trabajos se acumulan y tomamos la suma total como las horas trabajadas.

17 La entidad federativa se encuentra en la codificación de la variable FOLIO y para obtenerla sólo se busco la codificación en cada trabajador.
} 
introduce una variable dummy, en donde (1) se utiliza para hombres y (0) para mujeres. En el caso de la educación formal o escolaridad, se tuvo que hacer una unificación de criterios, ya que los juicios para codificar los niveles escolares en las tres encuestas fueron diferentes. Por tanto, la codificación utilizada fue la siguiente: 0 para trabajadores sin instrucción; 3 para trabajadores con primaria incompleta; 6 para trabajadores con primaria completa; 8 para trabajadores con secundaria incompleta; 9 para trabajadores con secundaria completa; 11 para trabajadores con preuniversidad incompleta; 12 para trabajadores con preuniversidad completa; 15 para trabajadores con superior incompleta; 17 para trabajadores con superior completa y 18 para trabajadores con estudios de posgrado ${ }^{18}$. La educación técnica las encuestas la dividen por niveles, es decir, la educación técnica que no requiere requisito para llevarla a cabo, la educación técnica que requiere de estudios de primaria para llevarla a cabo, la educación técnica que requiere de estudios de secundaria para llevarla a cabo y la educación técnica que requiere de estudios de pre-universidad para llevarse a cabo. La variable educación técnica, se utiliza para ver si la educación técnica tiene alguna influencia en los salarios por hora percibidos por los trabajadores, para ver esto, dividimos a los trabajadores entre aquellos que cuentan con educación técnica y los que no cuentan con educación técnica. Para lo cual, se utiliza una dummy, en donde (1) se utiliza para los trabajadores que cuentan

\footnotetext{
18 Cabe mencionar que si en cada caso se utilizaban los datos originales, los resultados para esta variable no varían en forma significativa de los que obtenemos con la nueva codificación.
} 
con educación técnica y (0) para los trabajadores que no cuentan con ella. En el caso de la variable rama, de nueva cuenta los criterios para clasificarlas no son los mismos en los tres años, pero en este caso no hay problema, pues procedimos a clasificar cada rama dentro del sector productivo primario, secundario y terciario ${ }^{19}$. Para utilizar esta información en la formulación, se crearon dos dummy. La primera, con (1) para el sector secundario y (0) para otros. La segunda, con (1) para el sector terciario y (0) para otros. Teniendo como variable de control al sector primario. En lo referente a la variable condiciones laborales, que tiene que ver con el tipo de contratación que tenía el trabajador al momento de ser encuestado (que es el tipo de contrato que tenía el trabajador con su patrón), a grandes rasgos en los tres años se puede dividir entre los trabajadores que tenían un contrato por escrito y los trabajadores que no tenían contrato o la contratación fue sólo de palabra. Para nuestros propósitos dividimos a los trabajadores entre aquellos que tenían un contrato por escrito -ya sea por tiempo determinado o indeterminado- y los trabajadores que no lo habían hecho, para lo cual, se utiliza una variable dummy, en donde (1) se utiliza para los trabajadores que tuvieron un contrato por escrito y (0) para otros. La variable sindicato, se refiere a si un trabajador está afiliado a un sindicato o no lo está. La variable sindicato, también se introducirá como una variable dummy, con el propósito de identificar el efecto

\footnotetext{
${ }^{19}$ En el sector primario, están las ramas de agricultura, ganadería, caza, silvicultura y pesca. En el sector secundario, están las ramas de minería y extracción de petróleo, industria manufacturera, electricidad y agua y construcción. En el sector terciario, comercio y servicios.
} 
que tiene sobre el salario del trabajador. Para realizar la variable dummy, el número uno (1), agrupará a aquellos trabajadores afiliados a un sindicato y la variable cero (0), agruparía el término otros. La variable entidad federativa, nos sirvió para dividir a los trabajadores por regiones. La primera región abarca a aquellos estados que antes de la apertura comercial, se consideraban los más industrializados, con mejores servicios, y por tanto, con mayor desarrollo. La segunda región abarca a aquellos estados que limitan con los Estados Unidos de Norteamérica y que como ya lo hemos mencionado, varios investigadores señalan como los estados que se verían beneficiados de forma directa con el Tratado de Libre comercio de Norteamérica y una tercera región que abarcaría a los restantes estados de la República Mexicana $^{20}$. En este caso, las variables dummy que se elaboran son dos. La primera, con (1) para los estados industrializados y (0) para otros. La segunda, con (1) para estados fronterizos y (0) para otros. La variable de control en este caso son los estados que no se incluyeron entre los estados fronterizos y los estados con mayor desarrollo industrial. La variable que hemos denominado educación formal (D_Ed_Formal), nos sirve para dividir al mercado de trabajo mexicano en dos, por un lado, estarán aquellos trabajadores con niveles oficiales de escolaridad formal básicos o sin escolaridad, y por otro, aquellos trabajadores con niveles de escolaridad oficiales superiores a los formales básicos. En el caso mexicano,

\footnotetext{
${ }^{20}$ La primera región abarca a las entidades federativas de Distrito Federal, Jalisco, Estado de México y Nuevo León. La segunda región a las entidades federativas de Baja California Norte, Coahuila, Chihuahua, Sonora y Tamaulipas. La tercera región abarca a las restantes entidades federativas de la República Mexicana.
} 
la división es casi natural, pues antes señalamos que el gobierno mexicano oficializó que la educación básica tendría una duración de nueve años, lo que en México se conoce como la educación primaria y secundaria, con lo cual, faltarían por estudiar de siete a ocho años, lo que en México se conoce como educación pre-universitaria, universitaria y posgrado. Por tanto, estaríamos dividiendo al mercado de trabajo entre aquellos trabajadores con niveles de educación de secundaria o menores (sin escolaridad o escolaridad básica) y aquellos trabajadores con educación superior a la básica (educación preuniversitaria, universitaria y posgrado).

Para estimar el modelo sólo se tomaron en cuenta a los trabajadores con edades comprendidas entre doce y sesenta y cinco años de edad. Esto se hace por dos razones: la primera, es que la afiliación a un sindicato en las encuestas, la puede hacer el trabajador desde los 12 años de edad y en segundo lugar, porque la edad de jubilación debería ser por lo regular a los 65 años de edad. Por último, cabe señalar que los modelos se procesaron utilizando el programa StataSE10.

\section{RESULTADOS}

El Cuadro 1, muestra los resultados de la combinación, mediante MCO e inferencia robusta. El modelo consta de 24, 450 trabajadores. Los datos entre paréntesis debajo de cada coeficiente son los errores estándares y el asterisco señala las variables que son 
significativas al 95\%. Cabe señalar que el estadístico $\mathrm{F}$ es significativo al $100 \%$.

Cuadro 1- Resultados de la combinación

\begin{tabular}{|c|c|}
\hline Variable & Coeficiente \\
\hline 1984 & $2.937511^{*}$ \\
& $(0.0474638)$ \\
\hline 1992 & $3.361997^{*}$ \\
& $(0.0581253)$ \\
\hline 2000 & $-2.193024^{*}$ \\
& $(0.0561085)$ \\
\hline Escolaridad-1984 & $0.0833487^{*}$ \\
& $(0.0043251)$ \\
\hline Escolaridad-1992 & -0.0018112 \\
& $(0.0052004)$ \\
\hline Escolaridad-2000 & -0.0044692 \\
& $(0.0051097)$ \\
\hline Experiencia-1984 & $0.04071077^{*}$ \\
& $(0.0026197)$ \\
\hline Experiencia-1992 & -0.0016064 \\
& $(0.003125)$ \\
\hline Experiencia-2000 & -0.0026131 \\
& $(-0.0026131)$ \\
\hline Experiencia2-1984 & $-0.0006241^{*}$ \\
& $(0.0000518)$ \\
\hline Experiencia2-1992 & 0.0000899 \\
& $(0.0000627)$ \\
\hline Experiencia2-2000 & $0.0001149^{*}$ \\
& $(0.0000608)$ \\
\hline Sexo-1984 & $0.1288776^{*}$ \\
& $(0.0225911)$ \\
\hline Sexo-1992 & $-0.0509138^{*}$ \\
& $(0.0268018)$ \\
\hline
\end{tabular}




\begin{tabular}{|c|c|}
\hline Sexo-2000 & $\begin{array}{c}-0.021847 \\
(0.0262839) \\
\end{array}$ \\
\hline Educación-Técnica-1984 & $\begin{array}{l}0.165939^{*} \\
(0.0244368)\end{array}$ \\
\hline Educación-Técnica-1992 & $\begin{array}{c}-0.0357793 \\
(0.0305197) \\
\end{array}$ \\
\hline Educación-Técnica-2000 & $\begin{array}{c}-0.132312^{*} \\
(0.0300303)\end{array}$ \\
\hline Contrato-1984 & $\begin{array}{c}0.4116404^{*} \\
(0.0228074)\end{array}$ \\
\hline Contrato-1992 & $\begin{array}{c}-0.182312^{*} \\
(0.0270827)\end{array}$ \\
\hline Contrato-2000 & $\begin{array}{r}-0.1157798^{*} \\
(0.0272681)\end{array}$ \\
\hline Sindicato-1984 & $\begin{array}{r}0.1730155^{*} \\
(0.0195533)\end{array}$ \\
\hline Sindicato-1992 & $\begin{array}{c}-0.0957189^{*} \\
(0.0252795)\end{array}$ \\
\hline Sindicato-2000 & $\begin{array}{l}0.0115514 \\
(0.0264403)\end{array}$ \\
\hline Sector-Secundario-1984 & $\begin{array}{c}0.4447824^{*} \\
(0.0351152)\end{array}$ \\
\hline Sector-Secundario-1992 & $\begin{array}{l}0.0237801 \\
(0.0420805)\end{array}$ \\
\hline Sector-Secundario-2000 & $\begin{array}{r}-0.2083754^{*} \\
(0.0411588)\end{array}$ \\
\hline Sector-Terciario-1984 & $\begin{array}{r}0.3919439^{*} \\
(0.0353762)\end{array}$ \\
\hline Sector-Terciario-1992 & $\begin{array}{l}0.0652025 \\
(0 . .0423843)\end{array}$ \\
\hline Sector-Terciario-2000 & $\begin{array}{c}-0.1704738^{*} \\
(0.0414237)\end{array}$ \\
\hline
\end{tabular}




\begin{tabular}{|c|c|}
\hline Estados-Industrializados- & $0.1607876^{*}$ \\
1984 & $(0.0198487)$ \\
\hline Estados-Industrializados- & $0.0909262^{*}$ \\
1992 & $(0.0237994)$ \\
\hline Estados-Industrializados- & $0.0619502^{*}$ \\
2000 & $(0.0251921)$ \\
\hline Estados-Fronterizos-1984 & $0.2163502^{*}$ \\
& $(0.0265204)$ \\
\hline Estados-Fronterizos-1992 & 0.0420693 \\
& $(0.033592)$ \\
\hline Estados-Fronterizos-2000 & $0.0745464^{*}$ \\
& $(0.0320134)$ \\
\hline Posible-Dualidad-1984 & $0.0746924^{*}$ \\
& $(0.036068)$ \\
\hline Posible-Dualidad-1992 & $0.1372806^{*}$ \\
& $(0.0436381)$ \\
\hline Posible-Dualidad-2000 & $0.0987029^{*}$ \\
& $(0.042577)$ \\
\hline Número de & 24450 \\
\hline Observaciones & 0.9495 \\
\hline R-Cuadrada & \\
\hline
\end{tabular}

Hay 4,344 trabajadores en la muestra de 1984, 10,396 en la de 1992 y 9,710 en la del año 2000. La intercepción de 1984 es $\beta_{0}=\ln Y_{0}=2.94$, la de 1992 es $\beta_{0}+\delta_{0}=6.3$ y la de $2000 \beta_{0}+\gamma_{0}=0.74$. Todas significativas. 
El efecto promedio de la escolaridad sobre el salario en 1984, se estima que es de $8.3 \%{ }^{21}$, el de 1992 es 0.18 puntos porcentuales menor al de 1984 y el del año 2000 es 0.45 puntos porcentuales menor al de 1984, es decir, el rendimiento de la escolaridad decrece en la apertura comercial. Pero como se puede observar en el cuadro, las reducciones en 1992 y 2000 son no significativas. En nuestro caso, diríamos que el impacto de la escolaridad sobre los salarios de los trabajadores, en la primera y segunda parte de la apertura comercial, no cambió en forma significativa, con respecto al que había en promedio en 1984. Una situación contraria, a la que señalaron numerosos investigadores para el caso de México. Antes señalamos que autores como Robertson (2001), habían mostrado que la tasa de retorno sobre escolaridad se incrementó de 1987 a 1994 y de 1994 a 1998. El atenuante que cabría en el trabajo de Robertson y otros, es que trabajaron con datos de empleo urbano, periodos diferentes o con otras encuestas, por lo que sus resultados son aceptables en este tipo de condiciones y para este tipo de trabajadores. En nuestro caso, creemos que este resultado refleja en mayor medida lo que sucede a los trabajadores que obtienen sus ingresos mediante remuneraciones al salario, en general, pues en el modelo se contemplan tanto a trabajadores urbanos como a trabajadores rurales.

Pero de cualquier manera, el resultado no parece $\tan$ intuitivo, si tomamos en cuenta que los niveles de escolaridad de estos trabajadores se han incrementado en

\footnotetext{
21 Por cierto, un rendimiento promedio que encuentran muchas de las investigaciones realizadas para México, como lo señala Villareal (2008).
} 
la primera y segunda etapa de la apertura comercial, es decir, en promedio los niveles escolares de estos trabajadores aumentaron en la primera etapa de la apertura comercial y aumentaron aún más en la segunda etapa de la apertura comercial ${ }^{22}$. De hecho si obtenemos el nivel salarial promedio para cada nivel escolar en los tres años, hay dos situaciones que son muy evidentes, la primera, es que en general, el salario promedio es mayor entre mayor es el grado de escolaridad alcanzado por el trabajador. La segunda, que en la primera parte de la apertura comercial, el salario promedio de los trabajadores con estudios no básicos aumentan en relación a los que tenían en 1984, y que en la segunda etapa de la apertura comercial es muy marcado el aumento en los salarios promedio de los trabajadores con estudios de posgrado. Entonces, la pregunta que nos tendríamos que hacer es ¿cuál es la causa por la que en promedio, el nivel escolar no ha tenido influencia en el salario de los trabajadores en la apertura comercial? La clave para explicar este fenómeno, la encontramos en el poder adquisitivo de los salarios y en la desigualdad salarial $^{23}$. La desigualdad salarial de los trabajadores se incrementó en la primera etapa de la apertura comercial y aún más en la segunda etapa. Para el año de 1984, la mayor desigualdad salarial, la encontramos entre los trabajadores sin escolaridad y la menor entre los trabajadores con posgrado. Para la primera parte de la apertura comercial, el índice se incrementa para todos los trabajadores con escolaridad y sólo decrece para los

\footnotetext{
22 Obteniendo los promedios de escolaridad, de las muestra que se utilizaron para correr el modelo.

${ }^{23}$ Para medir la desigualdad se utiliza el índice de Gini.
} 
trabajadores sin escolaridad. Pero cabe señalar que los índices se incrementan en mayor medida para trabajadores con estudios no básicos, en particular, llama la atención el aumento en el índice para los trabajadores con estudios de posgrado, su índice pasa de 0.19 en 1984 a 0.34 en 1992. Para la segunda etapa de la apertura comercial, el índice de los trabajadores sin escolaridad sigue cayendo. También caen los índices para los trabajadores con estudios secundarios y con licenciatura, aunque cabe decir, que aún son superiores a los que había en 1984. El índice para trabajadores con estudios de posgrado aumenta aún más, al pasar a 0.39. También aumentan los índices de los trabajadores con estudios de primaria y bachillerato. Los índices de estos últimos tres niveles escolares tienen una tendencia creciente y para los trabajadores sin escolaridad hay una tendencia decreciente. Aunque los niveles escolares aumentaron, también aumento la desigualdad salarial, es decir, sólo algunos trabajadores se han beneficiado de la relación positiva entre nivel escolar y salario.

Pero aunque la desigualdad es importante, el principal factor para que la educación no tenga efecto sobre los salarios de los trabajadores, es el poder adquisitivo de los mismos. En términos reales el poder adquisitivo de los salarios ha decrecido desde 1984. Según datos de la Secretaria del Trabajo y Previsión Social $^{24}$, el salario mínimo real diario a precios de 1994,

\footnotetext{
24 Salario Mínimo Real Promedio Nacional (pesos mexicanos de 1994), Estadísticas de la Comisión Nacional de Salarios Mínimos de la Secretaria del Trabajo y Previsión Social.
} 
en 1984, era de 24.56 pesos (mexicanos), en 1992, de 14.18 pesos y para el año 2000 , de 10.85 pesos. El salario real ha tenido una tendencia claramente decreciente, la política de atar el incremento de los salario a la tasa de inflación pronosticada por el gobierno federal, ha dado los frutos que de ella se esperaban. En este sentido, podemos calcular que el salario mínimo real de 1992, representaba el $57.74 \%$ del de 1984 y el salario mínimo real del año 2000, representaba el $44.18 \%$ del de 1984. El salario mínimo real ha perdido su poder adquisitivo desde antes de 1984, y aunque, los salarios nominales aumentaron con la escolaridad, en términos reales no se ha podido ver reflejado en promedio este efecto en las dos etapas de la apertura comercial.

Por lo que toca a la variable experiencia, en promedio tiene un impacto sobre la escolaridad de $4.1 \%$ en 1984, pero no es significativa para 1992 y 2000. Mientras que la experiencia al cuadrado, tiene el signo que se esperaba y un impacto sobre el salario de $-0.06 \%$, siendo no significativa en 1992 y significativa para el año 2000. El análisis de la experiencia es difícil en esta situación, pues para hacerlo, se deben tener en cuenta las dos variables. Para 1984, podemos decir que los años de experiencia para obtener los máximos salarios por hora en logaritmos naturales fueron 32 años con ocho meses, y dado que la experiencia no es significativa en los siguientes periodos, podemos decir que los años no varían significativamente en la primera y segunda etapa de la apertura comercial. 
Los resultados anteriores nos señalan, que la acumulación de capital humano mediante la escolaridad y la experiencia, no impactaron en promedio, en el salario por hora que gana un trabajador en México o podríamos decir, que estas dos variables en promedio, tienen una importancia menor, entre los factores que determinan el salario por hora de los trabajadores en México.

El efecto promedio de la variable sexo sobre el salario en 1984, es de $12.9 \%$. Es decir, en promedio los hombres (ceteris paribus), antes de la apertura comercial, recibían un salario $12.9 \%$, mayor al que recibían las mujeres, por realizar las mismas tareas. Lo que se puede traducir en que hay una discriminación laboral hacia la mujer en este año. Para 1992, el efecto es 5.1 puntos porcentuales menor al de 1984, es decir, el efecto cae a $7.8 \%$, lo que se podría considerar, como un decrecimiento de la discriminación laboral. En la segunda etapa de la apertura comercial, el efecto no es significativo, con respecto a 1984. Esto último nos estaría indicando que para este año, la discriminación volvió a niveles similares a los que había antes de la apertura comercial. Un dato aún más preocupante, si consideramos que la participación de la mujer en el mercado laboral mexicano, aumentó en $5.7 \%$ de 1984 al año 2000.

El efecto promedio de la educación técnica sobre el salario (ceteris paribus) fue de $16.6 \%$ en 1984. Antes de la apertura comercial, los trabajadores con este tipo de educación, en promedio obtenían un salario $16.6 \%$ 
mayor, al que reciben los trabajadores sin este tipo de educación. El cambio para la primera etapa de la apertura comercial, no fue significativo, por lo que podríamos decir que las condiciones de 1984 se mantuvieron. Para la segunda etapa de la apertura comercial hay un efecto de 13.2 puntos porcentuales menor al de 1984, es decir, para el año 2000, el efecto baja notablemente, para ubicarse en 3.4\%. Para este año, un trabajador con educación técnica, en promedio, obtienen un salario $3.4 \%$ mayor, al que reciben los trabajadores sin este tipo de educación. Antes señalamos que el gobierno tenía un enorme interés en la vinculación de la escolaridad y el puesto laboral. Y uno de los ejes para hacerla, era la educación técnica. En su momento, el gobierno aposto mucho por este tipo de educación, pensando que ella incidiría directamente en la productividad del trabajador, en beneficio de la empresa y de los salarios de los trabajadores. Pero el panorama para los trabajadores con este tipo de estudios, se ensombreció en la segunda etapa de la apertura comercial.

El caso de la variable contrato, es muy llamativo, pues es una de las variables que en promedio, influye en mayor medida en el salario en estos tres años. El efecto promedio de esta variable sobre el salario antes de la apertura comercial era de $41.2 \%$. Un trabajador con contrato por escrito (ceteris paribus), en promedio, obtenía un salario $41.2 \%$ mayor. Para la primera etapa de la apertura comercial, se tiene un efecto de 18.2 puntos porcentuales menor al de 1984, es decir, para 1992, un trabajador con contrato por escrito, ganaba $23 \%$ más, 
que un trabajador sin contrato por escrito. La influencia de esta variable decae en la primera etapa. Para la segunda etapa de la apertura comercial, el efecto es de 11.6 puntos porcentuales menores al de 1984. Es decir, para el año 2000, un trabajador con contrato por escrito obtenía un salario $29.6 \%$ mayor. El salario promedio de los trabajadores con contrato por escrito repunta en la segunda etapa de la apertura comercial, aunque ya no tiene la importancia que tuvo antes de la apertura. Este fenómeno creemos, tiene que ver, con la subcontratación y del empleo informal ${ }^{25}$, pues los trabajadores sin contrato por escrito, aumentan en la apertura comercial. En términos generales, un trabajador con contrato por escrito y que por consecuencia, en la mayoría de los casos, cuenta con prestaciones de ley, es lo que se conoce como un trabajador formal. Por tanto, estaríamos señalando que los trabajadores formales, ganan salarios por hora mayores, a los que ganan trabajadores que no están en esta categoría, aunque cabría decir, que en la apertura comercial la brecha entre unos y otros se ha ido reduciendo. En este mismo sentido, cabe señalar que en los tres años, los trabajadores que no contaban con contrato por escrito, en promedio tenían menos años de escolaridad y se ubicaban principalmente en los sectores agropecuarios y de construcción. Siendo, como cabria

\footnotetext{
25 La subcontratación es el proceso económico en el cual una empresa determinada mueve o destina los recursos orientados a cumplir ciertas tareas, a una empresa externa, por medio de un contrato. Las empresas externas que contratan empleados en México, en la mayoría de los casos, los contratan por medio de lo que se conoce como pago por honorarios. Una contratación que no crea vínculos laborales, ni prestaciones. Por tanto el empleador, puede dar de baja al trabajador en el momento que lo decida, sin tener que pagarle nada. Recordemos que en México no existe seguro de desempleo a nivel federal.
} 
esperar las principales ocupaciones de estos trabajadores, las de trabajadores en el proceso agropecuario, obreros, operadores y artesanos. Aunque, también es importante señalar que las ocupaciones de servicio doméstico, operadores de transporte y vendedores ambulantes, aumentaron en la apertura comercial entre los trabajadores sin contrato por escrito.

En cuanto a la variable sindicato, lo primero que debemos tener en cuenta, es que, en la apertura comercial, el número de trabajadores afiliados a algún sindicato decae drásticamente, pasan de $24.5 \%$ en 1984 a 16.4 en el año 2000. Antes de la apertura comercial un trabajador sindicalizado ganaba (ceteris paribus) en promedio $17.3 \%$ más, de lo que lo hacía un trabajador no sindicalizado. En la primera etapa de la apertura comercial, se tiene un efecto de 9.6 puntos porcentuales menor al de 1984. Es decir, en 1992, un trabajador afiliado a un sindicato obtenía un salario promedio 7.7\% mayor, al que recibía un trabajador no afiliado. La importancia que tiene la defensa de los salarios por parte de los sindicatos decae en promedio en esta primera etapa, esto puede ser el reflejo, del pacto que hicieran los sindicatos con líderes empresariales y el gobierno, para amarrar la negociación salarial a la inflación pronosticada por el gobierno en turno. Para la segunda etapa de la apertura comercial, el efecto no es significativo, es decir, la variación no es significativamente diferente a la que había en 1984. Para esta etapa, los sindicatos recuperaron, de algún modo, su papel en la defensa de los salarios de los trabajadores afiliados a ellos. En este caso, pensamos que los 
sindicatos más débiles desaparecieron y sobrevivieron los sindicatos poderosos, que desde antes de la apertura comercial tenían altas prestaciones y salarios, como son los sindicatos de la educación, de los electricistas, de los petróleos y otros. Al desaparecer los sindicatos más débiles, con menores prestaciones y salarios, el efecto de los sindicatos poderosos con mayores salarios, se ve reflejado en un aumento promedio de los salarios de los trabajadores afiliados a algún sindicato, en esta segunda etapa de la apertura comercial.

Para el caso de la variable sector productivo, debemos señalar, en primer lugar, que se escogió como variable control al sector primario, por ser en México, el sector que en términos generales, se puede señalar contiene una gran cantidad de procesos productivos atrasados, y en donde, se concentra una parte muy importante de la población más marginada en el país. Por ello, no es sorprendente que antes de la apertura comercial, las diferencias salariales con los trabajadores de los otros sectores sean tan marcadas. Antes de la apertura comercial, los trabajadores del sector secundario ganaban (ceteris paribus) en promedio $44.5 \%$ más, que los trabajadores del sector primario y los trabajadores del sector terciario 39.2\% más, que los trabajadores del sector primario. En la primera etapa de la apertura comercial, la variación porcentual en el sector secundario y terciario respecto a 1984, es no significativa, es decir, las condiciones que había en 1984, se mantienen sin cambios significativos para 1992. Para la segunda etapa de la apertura comercial, se encuentra un efecto de 20.8 puntos porcentuales menor 
al de 1984, para el sector secundario y de 17 puntos porcentuales menor respecto al de 1984, para el sector terciario. Es decir, para el año 2000, los trabajadores del sector secundario, ganaban en promedio $23.7 \%$ más que los trabajadores del sector primario y los trabajadores del sector terciario, ganaban en promedio $22.2 \%$ más que los trabajadores del sector primario. Ambos porcentajes se reducen en alrededor de la mitad de los que habían en 1984, la brecha salarial se acorta en esta segunda etapa. Esto último pensamos, se puede deber a que en porcentajes totales, la cantidad de trabajadores en el sector primario ha disminuido, aumentando la oferta de trabajadores en los sectores secundario y terciario con el consecuente efecto que esto tiene sobre los salarios de estos trabajadores. Además, se debe tener en cuenta, que los diferentes gobiernos mexicanos, desde los años ochentas, no han creado las condiciones, para que la economía mexicana genere los empleos necesarios para los individuos que se incorporan al mercado laboral, por lo que, hay un déficit de puestos de trabajo y una oferta que aumenta, empujando los salarios reales a la baja. En este sentido, otra situación que puede explicarlo, es que algunos trabajadores del sector secundario y terciario han pasado al sector informal en donde los salarios pagados son menores. Por otra parte, un aspecto que se puede observar, es que el salario por hora promedio de los trabajadores del sector secundario, se mantiene siempre por arriba del salario por hora promedio del sector terciario, en términos del salario por hora promedio del sector primario, es decir, el orden no cambia en la apertura comercial, los salarios por hora promedios más altos son los del sector secundario, 
seguidos de los del sector terciario y por último los del sector primario.

En el caso de la variable entidad federativa. La variable control en este caso fue otros, pues esperábamos ver los cambios promedio que en los salario tendría la región en donde habitaban los trabajadores, especialmente en las entidades fronterizas y en las entidades que hemos denominado industrializadas. Como lo han señalaron otros trabajos de investigación que hemos mencionado al inicio, en la apertura comercial, los trabajadores que laboraban en estados fronterizos y los que denominamos industrializados se vieron favorecidos en sus salarios por hora con respecto a otros. Antes de la apertura comercial, los salarios promedio (ceteris paribus) de los trabajadores que laboraban en los estados denominados industrializados, eran $16.1 \%$ mayores, que los salarios de los trabajadores que laboraban en las denominadas otras entidades. En la primera etapa de la apertura comercial, se encuentra un efecto de 9.1 puntos porcentuales mayor al que había en 1984, es decir, para el año de 1992, los trabajadores que laboraban en los estados denominados industrializados, en promedio ganaban $25.2 \%$ más, que los trabajadores que laboraban en otros. Y para la segunda etapa de la apertura comercial, encontramos un efecto de 6.2 puntos porcentuales mayor al que había en 1984, es decir, para el año 2000, los trabajadores que laboraban en los estados denominados industrializados, en promedio ganaban $22.3 \%$ más, que los trabajadores que laboraban en otros. Aunque, el efecto positivo, disminuye un poco en la segunda etapa de la apertura comercial, parece que 
es evidente el efecto promedio positivo que tiene la apertura comercial sobre los salarios de los trabajadores que laboraban en los estados que hemos denominado industrializados. Esto concuerda con la hipótesis de que con la apertura comercial, se iban a beneficiar estos estados, por tener los servicios e infraestructura más desarrollada, en dos sentidos: muchas empresas se ubicarían en estos estados para aprovechar estas dos ventajas y las empresas ubicadas fuera de estos estados, tendrían que utilizar estas ventajas para poder competir a nivel internacional.

Por otro lado, antes de la apertura comercial, un trabajador que laboraba en los estados fronterizos, ganaba (ceteris paribus) en promedio $21.6 \%$ más, que un trabajador de los estados denominados otros. Para la primera etapa de la apertura comercial, la variación porcentual en los estados fronterizos respecto a 1984, es no significativa, es decir, las condiciones que había en 1984, se mantienen sin cambios significativos para 1992. En cambio, para la segunda etapa de la apertura comercial, se encuentra un cambio positivo porcentual de 7.5. Es decir, en el año 2000, un trabajador de un estado fronterizo ganaba en promedio $29.1 \%$ más, que un trabajados de los estados denominados otros. Esto también concuerda con los resultados obtenidos por otros investigadores -algunos de los cuales hemos mencionado al inicio de este trabajo-, en el sentido que los principales beneficiarios de un tratado con los E.U. y Canadá, serían los estados fronterizos, por tener México su mayor volumen comercial, precisamente con los E.U., que es lo que precisamente se nota en este último 
resultado, el salario se incrementa significativamente hasta la segunda etapa de la apertura comercial.

Por último, se debe señalar que, desde antes de la apertura comercial, los salarios promedio en los estados fronterizos $y$ en los que hemos denominado industrializadas, eran mayores a los que había en los estados no incluidos en estas dos categorías. Por tanto, se puede decir, que el efecto que se ha producido con la apertura comercial, es la agudización promedio de esas diferencias salariales, en perjuicio de los trabajadores que no pertenecen a estos estados.

La última variable utilizada en el modelo, es la que hemos denominado D_Ed_Formal. Una variable que hemos utilizado para dividir a los trabajadores entre aquellos que cuentan con escolaridad básica o sin escolaridad y los trabajadores con escolaridad mayor a la básica, con el propósito de encontrar indicios de alguna división institucional en el mercado de trabajo mexicano que afecte su salario ${ }^{26}$. En los resultados anteriores hemos encontrado algún indicio de esta división y también hallamos algunos indicios en el análisis de datos que se han efectuado para las bases de datos y en

\footnotetext{
26 Denominamos división institucional, a aquella división que fue propiciada por las políticas estatales y no por el mercado. En nuestro caso una división al interior del mercado laboral mexicano, propiciada por la política escolar, de dividir a los trabajadores entre los que cuentan con educación básica (que es obligatoria y gratuita) y los trabajadores con educación superior a la básica (que ya no es obligatoria y en general, ya no es gratuita). Una división que creemos se acentúo y se puede consolidar con la apertura comercial.
} 
especial para las variables utilizadas ${ }^{27}$. Antes de la apertura comercial, los trabajadores con estudios superiores a los básicos, en promedio (ceteris paribus) obtenían unos salarios $7.5 \%$ mayores, a los que recibían los trabajadores con escolaridad básica o sin escolaridad. En la primera etapa de la apertura comercial, hay un cambio porcentual positivo de 13.7 , con respecto a 1984 , es decir, para el año de 1992, los trabajadores con estudios superiores a los básicos ganan en promedio $21.2 \%$ más. Para la segunda también hay un cambio porcentual positivo, pero menor al de 1992, de 9.9, con respecto al que había en 1984, es decir, para el año 2000, los trabajadores con estudios superiores a los básicos, obtienen ingresos promedio $17.4 \%$ superiores. En la primera etapa de la apertura comercial, la influencia de este factor, aumenta un poco menos del triple. Y, aunque, el porcentaje cayó en la segunda etapa de la apertura comercial, aún así, este porcentaje, es muy superior al porcentaje que había antes de la apertura comercial (más del doble). Podemos hablar, de que con la apertura comercial, hubo un premio salarial a los trabajadores con estudios superiores a los básicos.

\section{CONCLUSIONES}

\footnotetext{
${ }^{27}$ Luyando, José R. (2009), "Factores que han Influido en la Determinación de la Tasa Salarial en el Mercado de Trabajo Mexicano en Tres Periodos: 1984 (Antes de la Apertura Comercial), 1992 (Con el Ingreso de México al GATT) y 2000 (Con el Ingreso de México al TLC)”, trabajo in extenso inédito.
} 
Cuando se incluyen en el análisis a todos los trabajadores que obtienen ingresos netos por remuneraciones al salario, encontramos que el retorno sobre escolaridad no tiene cambios significativos promedio en la apertura comercial. Lo mismo sucede cuando consideramos la experiencia. Un resultado que resulta interesante, en el sentido, de que muchos trabajos dan por hecho un efecto positivo, entre escolaridad y salarios en la apertura comercial. En el presente trabajo, encontramos que el efecto no ha sido uniforme, y mientras unos trabajadores se han beneficiado más de esta relación, otros tantos no lo han hecho, es decir, pueden haber enormes diferencias en salarios entre trabajadores que ostentan el mismo nivel escolar y este efecto es más notorio en los trabajadores con estudios superiores a los básicos en la apertura comercial.

La discriminación de género, disminuye en la primera etapa, pero vuelve a los niveles de antes de la apertura para el año 2000, aún y cuando en el TLC, se firma un acuerdo paralelo denominado Acuerdo de Cooperación Laboral par América del Norte, en donde uno de los puntos es precisamente combatir ésta. La educación técnica, pierde importancia entre los factores que afectan positivamente al salario, aún y cuando el gobierno mexicano apostó por este tipo de educación para elevar la productividad de los trabajadores y de este modo incidir en las ganancias de las empresas. Los trabajadores que describimos como formales en promedio ganan un mayor salario que los trabajadores que describimos como informales, aunque esta brecha disminuye en la segunda etapa de la apertura comercial. 
El porcentaje de trabajadores sindicalizados ha disminuido y esto pensamos se ha reflejado en la cantidad y tamaño de los sindicatos, y por tanto, de su capacidad para negociar salarios. Pensamos que este efecto derivó en el cierre de muchos pequeños sindicatos y la sobrevivencia de los más grandes, que intuimos pudo derivar en mayores salarios promedio para los trabajadores afiliados a estos sindicatos, lo cual, se vería reflejado en la segunda etapa de la apertura comercial. Desde antes de la apertura comercial, los trabajadores del sector terciario y secundario, ganaban salarios promedio mayores a los que obtenían los trabajadores del sector primario, lo llamativo en este caso, es que la brecha disminuye en la segunda etapa de la apertura comercial, una posible causa de este fenómeno pensamos se debe a que disminuye el porcentaje de trabajadores en el sector primario y aumenta en el sector secundario y terciario, es decir, aumenta la oferta en estos dos sectores, lo que indudablemente afecta sus salarios. Otra posible causa de esta disminución, pensamos se debe al aumento de los trabajadores informales en los sectores secundario y terciario. También queda confirmado el efecto que tuvieron los tratados de libre comercio sobre los salarios de los trabajadores de los estados colindantes con los Estados Unidos de Norteamérica, sus salarios tienen una tendencia creciente con respecto a los trabajadores de otros estados de la República, aunque hay que tener en cuenta que desde antes de la apertura comercial los salarios de estos trabajadores, eran en promedio superiores a los que había en otros estados de la República. Por último, debemos señalar que hay algún 
indicio de una segmentación en el mercado laboral, que llamamos institucional, pues fue propiciada por la misma división que hace el gobierno en los años de estudio. Una hipótesis que se tendría que confirmar o rechazar al hacer un estudio más completo del tema.

Por último, creemos que hay evidencia para sostener que la contención salarial realizada por el gobierno, al atar los aumentos salariales a la inflación esperada, ha tenido influencia en todos los resultados que hemos obtenido. Por lo que, estamos de acuerdo con lo que señala Feliciano (2001), en el sentido que la apertura comercial tuvo un modesto impacto en el mercado laboral mexicano y que los mayores efectos en los salarios de los trabajadores mexicanos se debe principalmente al programa de estabilización económica denominado Pacto de Solidaridad Económica.

\section{REFERENCIAS BIBLIOGRAFICAS}

Adsera, A. y Boix, C., (1998), Must We Choose? European Unemployment, American Inequality and the Impact of Education and Labor Market Institutions, W.P.414.98, Discussion Papers, Departament d'Economia $i$ d'História Económica e Institut d'Análisi Económica, CSIC. Barceinas, F., (2003), "Endogeneidad y Rendimientos de la Educación", Estudios Económicos 35, Vol. 18, $\mathrm{N}^{\circ}$ 1, Enero-Junio, pp. 79-131. 
Becker, G., (1962), "Investment in Human Capital: A Theoretical Analysis" Journal of Political Economy, Vol. 70, Issue S5, page 9, Jan.

Becker, G., (1964), Human Capital: A Theoretical and Empirical Analysis whit Special Reference to Education, National Bureau of Economic Research, New York.

Bernan E., Bound J. and Griliches, Z., (1994), "Changes in the Demand for Skiller Labour within U.S. Manufacturing: Evidence from the Annual Survey of Manufacturers", The Quarterly Journal of Economics, Vol. 109, $\mathrm{N}^{\circ} 2$, May, pp. 367-397.

Berndt, E,. (1991), The Practice of Econometrics:

Classic and Contemporary, ed. Addison Wesley, United States of America.

Blackburn, M., Bloom, D. and Freeman, R. (1990/91), "An Era of Falling Earning and Rising Inequality", Brookings Review, Vol. 9, $\mathrm{N}^{\circ} 1$ Winter, pp. 38-43.

Bluestone, B. and Harrison B., (1988), The Great UTurn: Corporate Restructuring and Polarization of America, Ed. Basic Books, New York.

Borjas, G., Freeman R. and Katz L., (1996), "Searching for the Effect of Immigration on the Labor Market", American Economic Review, May, pp.246-251.

CD-ROM, Encuesta Nacional de Ingreso Gasto de los Hogares (ENIGH), INEGI, México 1984.

CD-ROM, Encuesta Nacional de Ingreso Gasto de los Hogares (ENIGH), INEGI, México1992. 
CD-ROM, Encuesta Nacional de Ingreso Gasto de los Hogares (ENIGH), INEGI, México 2000.

Cragg, M. and Epelbaum, M., (1996), "Why Has Wage Dispersion Grow in Mexico? Is It the Incidence of Reforms or the Growing Demand for Skills?", Journal of Development Economics, Vol. 51, $\mathrm{N}(1)$, October, pp. 99-116.

Davis, S. and Haltiwaanger, J., (1991), "Wage Dispersion Between and Within U.S. Manufacturing Plants, 1963-86", Brookings Papers on Economic Activity, Microeconomics, Vol. 1991, The Brookings Institution, pp. 115200.

Feenstra, R. and Hanson, G., (1997), "Foreign Direct Investment and Relative Wages: Evidence from Mexico's Maquiladoras", Journal of International Economics, Vol. 42, May, pp. 371393.

Feliciano, Z., (2001), "Workers and Trade Liberalization: The Impact of Trade Reforms in Mexico on Wages and Employment", Industrial and Labor Relations Review, Vol. 55, (1), Oct, pp. 95-115.

Griliches, Z.. (1977), "Estimating the Returns to Schooling: Some Econometric Problems", Econometrica, Vol. 45, № 1, January, pp. 1-22.

Hanson, G., (2005), "Globalization, Labor Income and Poverty in Mexico", NBER Working Papers Series 11027, Cambridge, MA, January, p. 52.

Hanson, G., (2003), "What has Happened to Wages in Mexico since NAFTA? Implications for 
Hemispheric Free Trade", NBER Working Papers Series 9563, Cambridge, MA, p. 45.

Hanson, G. and Harrison, A., (1995), "Trade Technology and Wage Inequality", NBER Working Papers Series 5110, Cambridge, MA, pp. 271-288.

Hanson, G. and Harrison, A., (1999), "Trade Liberalization and Wage Inequality in Mexico", Industrial and Labor Relations Review, Vol. 52, (2), January, pp. 271-288.

Heckman, J. and Polachek, S., (1974), "Empirical Evidence on Functional Form of EarningsSchooling Relationship", Journal of the American Statistical Association, Vol. 69, (346), Jun, pp. 350-354.

Juhn, C., Murphy, K. and Pierce, B., (1993), 'Wage Inequality and the Rise in Returns to Skill', Journal of Political Economy, number 3, Volume 101, The University of Chicago Press, June, pp. 410-442.

Katz, L. and Murphy, K. (1992), "Changes in Relative Wages in the United States: Supply and Demand Factors", Quartely Journal of Economics, Vol. 107, (1), Feb., pp. 35-78.

Katz, L. and Rebenga, A., (1989), "Changes in the Structure of Wages: The United States vs Japan", Journal of the Japanese and International Economies, December, pp. 522-553.

Krueger, A., (1993), "How Computers Have Changed the Wage Structure: Evidence from Microdata, 1984-1989", The Quarterly of Journal of Economics, Vol. CVIII, (1), February, pp. 33-61. 
Lawrence, R. and Slaughter, M., (1993), "International Trade and American Wages in the 1980s: Gaint Sucking Sound or Small Hiccup", Booking Papers on Economic Activity, Microeconomics, (2), pp. 161-226.

Luyando, J., (2007), Ensayos Sobre Mercados de Trabajo Duales: El Caso Mexicano, Tesis Doctoral, Departamento del Análisis Económico, Historia e Instituciones Económicas, Universidad de Valladolid.

Luyando, J., (2009), Factores que han Influido en la Determinación de la Tasa Salarial en el Mercado de Trabajo Mexicano en Tres Periodos: 1984 (Antes de la Apertura Comercial), 1992 (Con el Ingreso de México al GATT) y 2000 (Con el Ingreso de México al TLC), Trabajo de investigación posdoctoral en la Universidad Autónoma Metropolitana Iztapalapa, en el doctorado en Estudios Sociales.

Kosters, M., (1990), "Schooling, Work Experience and Wage Trends", The American Economic Review, Vol.80, (2), May, pp. 308-312.

Mincer, J., (1957), A Study of Personal Income Distribution, unpublished Ph.D. dissertation, Columbia University, Department of Economics, New York, USA.

Mincer, J., (1958), "Investment in Human Capital and Personal Income Distribution", Journal of Political Economy, August, p. 281.

Mincer, J., (1962), "On-the-Job Training: Costs, Returns and Some Implications", Journal of Political Economy, Vol.70, 5, October, pp. 50-79. 
Mincer, J., (1974), Schooling, Experience and Earnings, Columbia University Press, National Bureau of Economic Research, New York.

Mincer, J. and Polachek, S., (1974), "Family Investment in Human Capital: Earnings of Women", Journal of Political Economy, March-April, pp. 76-108.

Murphy, K. And Welch F. (1991), "Wage Premiums for College Graduates: Recent Growth and Possible Explanations", Educational Researcher, Vol. 18, (4), May, pp. 17-26.

Rebenga, A., (1997), "Employment and Wages Effects of Trade Liberalization: The Case of Mexico Manufacturing", Journal of Labor Economics, Vol. 15, (3), July, pp. 20-43.

Robertson, R., (2004), "Relative Prices and Wage Inequality: Evidence from Mexico", Journal of International Economics, Vol.64, dec., pp. 387409.

Shultz, T., (1960), "Capital Formation by Education", The Journal of Political Economy, Vol. 68, p.571.

Shultz, T., (1961), "Investment in Human Capital", The American Economic Review, Vol. 51, (1), March, pp. 1-17.

Slaughter, M., (1999), "Globalisation and Wage: A Tale of Two Perspectives", The World Economy, Blackwell Publishers, Vol. 22, (5), July, pp. 609629.

Stolper, W. and Samuelson, P., (1941), "Protection and Real Wages", Review of Economic Studies, Vol. 9, (1), Nov., pp. 58-73. 
Urciaga, J. y Almendarez, M., (2006), "Determinación de los Salarios y Rendimientos de la Escolaridad en la Región Mar de Cortés", Revista de la Educación Superior, abril-junio, Vol. XXXV (2), (138), pp. 37-53.

Villarreal, Edna M., (2008), "Evolución Histórica de los Rendimientos Educativos en México 19872004”, Estudios Sociales, CIAD, Vol. XVI, (32), Julio-Diciembre, pp. 59-78.

Wooldridge, J., (2002), Introducción a la Econometría: Un Enfoque Moderno, Ed. Thomson Learning, México. 